\title{
Damage detection of cracks in carbon fibre reinforced composites by pulsed eddy-current testing
}

\author{
Abdeslam Aoukili ${ }^{1, a}$ and Abdellatif Khamlichi ${ }^{2}$ \\ ${ }^{1}$ Department of Physics, Faculty of Sciences at Tetouan, University Abdelmalek Essaadi, Tetouan 93030, Morocco \\ ${ }^{2}$ Department STIC, National School of Applied Sciences at Tetouan, University Abdelmalek Essaadi, Tetouan 93030, Morocco
}

\begin{abstract}
Non-destructive evaluation techniques that are based on electromagnetic methods are commonly used for inspection of metallic and carbon fibre reinforced plastics parts. Some of these inspection methods are based on eddycurrents which are generated by the electromagnetic induction phenomenon occurring under variable magnetic induction field. In eddy-currents testing, the depth of penetration into the material is controlled by the conductivity of the tested material and also the work frequency. Transient eddy-currents, also called pulsed eddy-currents, is an emerging non destructive technique that employs a pulsed excitation to induce a transient electromagnetic response from defects lying deep within a conducting structure. Such defects are difficult to inspect by conventional techniques, such as harmonic eddy-currents or ultrasonics. A large number of recent scientific publications have dealt with the theoretical understanding of the pulsed eddy-currents phenomenon and have also undertaken the design feature of appropriate probes. Finite element solution of the governing equations has been used to simulate the output signals as function of the input electrical excitation signal. Considering a B-scan strategy, simulation of a pulsed eddy-currents based probe is performed in this work with the objective to assess detectability of small defects through monitoring impedance changes of a detection probe.
\end{abstract}

\section{Introduction}

Composites made from Carbon Fiber Reinforced Plastics (CFRP) have superior material properties in comparison with metallic components, such as: light weight, robustness, damage tolerance, fatigue strength and corrosion resistance. CFRP based composites are being increasingly used as structural components in many industrial applications such as aerospace, shipping and automotive. However, due to their permanent exposure to external forces resulting from impact events or to the action of alternating loading, the performance of composite structures can be harmfully alterated. Defects, either resulting from manufacturing or in-service induced damage cannot be always identified by simple visual inspection. Therefore, various non-destructive testing (NDT) techniques have been used to detect and evaluate defects and damage. These include acoustic emission, ultrasonic testing, eddy current, thermography, shearography, X-ray, ultrasonic waves,...

Non-destructive evaluation (NDE) techniques based on electromagnetic methods have been commonly used for inspection of metallic parts [1]. Some of these inspection methods are based on eddy-current (EC) technique. EC are a consequence of electromagnetic induction phenomenon taking place in a conductive medium. In eddy current testing, a probe carrying varying current is placed in close proximity to the material to produce a variable electromagnetic field, and then the eddy currents are generated within the inspected materials in response to the incident electromagnetic field. These currents form closed loop circuits within the inspected material and generate in their turn magnetic fields, in the way that they oppose the effects of the applied electromagnetic field.

For damage detection in CFRP composites EC testing (ECT) has been use [2]. The authors have used rotary probes to detect fibre orientation without lateral probe movement. They have found that the proposed eddycurrent scanning system is able to visualise fibre orientation, local imperfections like fibre fraction fluctuation, resin rich zones, delaminations and impact damages.

When EC are produced in a conductive material, they are not distributed uniformly throughout the inspected object. Instead, they tend to remain concentrated near to the sample surface and become less intense as the distance away from the surface increases. At a certain distance from the surface, EC effect becomes negligible and can be ignored. This phenomenon is called the skin effect which limits the depth of penetration of EC. The depth of penetration into the tested material is controlled by the conductivity of the material and also the work frequency. Transient eddy-current (TEC), also called pulsed eddy-current (PEC), is an emerging NDT technique that employs a pulsed excitation to induce a

\footnotetext{
$\overline{{ }^{a} \text { Corresponding author: aoukilia@yahoo.fr }}$
} 
transient electromagnetic response from defects lying deep within a conducting structure. Such defects are difficult to inspect by conventional techniques, such as harmonic eddy-currents or ultrasonics. A large number of recent scientific publications have dealt with the theoretical understanding of the TEC phenomenon and have also undertaken the design feature of appropriate probes [3-6].

To analyse response of TEC probes in terms of input and output signals, Finite Element (FE) simulation of the governing equations was considered [7].

Considering a B-scan strategy which consists in moving a probe along a straight path, the FE modelling under Comsol Multiphysics software packages is considered in the present work. A probe compound by two coils: inductive and receiver is analyzed under TEC excitation. The objective is to study delectability of a small defect through monitoring impedance changes of detection probe.

\section{Materials and method}

The constitutive equations governing a perfect Ohmic homogeneous and isotropic conductive medium write

$$
\left\{\begin{array}{l}
\vec{J}=\sigma \vec{E} \\
\vec{D}=\varepsilon \vec{E} \\
\vec{B}=\mu \vec{H}
\end{array}\right.
$$

where $\vec{E}$ is the electric field, $\vec{B}$ the magnetic induction field, $\vec{D}$ the electric displacement field, $\vec{H}$ the magnetic field, $\varepsilon$ the permittivity, $\mu$ the permeability and $\sigma$ the conductivity.

In case of a good conductive material such as a metal or CRFP, it is expedient to assume $\rho=0$ in Maxwell equations. A decoupling happens then and the following propagation-diffusion equations are readily obtained

$$
\left\{\begin{array}{l}
\Delta \vec{E}-\varepsilon \mu \frac{\partial^{2} \vec{E}}{\partial t^{2}}-\mu \sigma \frac{\partial \vec{E}}{\partial t}=\overrightarrow{0} \\
\Delta \vec{B}-\varepsilon \mu \frac{\partial^{2} \vec{B}}{\partial t^{2}}-\mu \sigma \frac{\partial \vec{B}}{\partial t}=\overrightarrow{0}
\end{array}\right.
$$

The magnetic permeability $\mu$ of CFRP composite material is equal to that of the free space $\mu=4 \pi \times 10^{7} \mathrm{H} / \mathrm{m}$ as the CFRP is nonmagnetic. The CFRP composites cannot be well described by the permittivity $\varepsilon$ which is of order $10^{-11} \mathrm{~F} / \mathrm{m}$, as they are conductive rather than dielectric. The conductivity is in general greater than $20 \mathrm{~S} / \mathrm{m}$ and can reach $2000 \mathrm{~S} / \mathrm{m}$. Considering low work frequencies not exceeding $10^{3} \mathrm{~Hz}$ one can verify that the propagative terms are negligible. This enables to simplify equations (2) by neglecting the propagation terms and keeping just the diffusive parts as follows

$$
\left\{\begin{array}{l}
\Delta \vec{E}-\mu \sigma \frac{\partial \vec{E}}{\partial t}=\overrightarrow{0} \\
\Delta \vec{B}-\mu \sigma \frac{\partial \vec{B}}{\partial t}=\overrightarrow{0}
\end{array}\right.
$$

Equations (1) and (3) yield the existence of a permanent density of currents that are circulating in the conductive material and having the form $\vec{J}=\sigma \vec{E}$, these compose the EC. For a given specimen geometry, the field $\vec{J}$ can be computed by solution of equations (3) in a given domain under specified boundary conditions.

In the presence of a singularity or a flaw in the medium, the conductivity varies and the EC distribution will show changes as compared to the pattern associated to the intact geometry. This provides a way for using EC as indicators to monitor flaw detection in CFRP made parts.

The practical implementation of EC technique for diagnosis purpose of flaws sets usually on two circuits. The first one constitutes the inductive part which is excited by a current pulse centred on a given work frequency as that shown in Figure 1. This puts variable magnetic field in the test material. The second circuit takes the form of a single coil or multiple coils sensor. Both circuits are made from metal like copper. Figure 2 depicts the EC probe proposed in this work. It consists of two separate coils. These coils are influenced by the variable magnetic field created by the EC inside the test material. The receiver coil undergoes impedance variation induced by the EC when the excitation coil is crossing a zone with variable local conductivity.

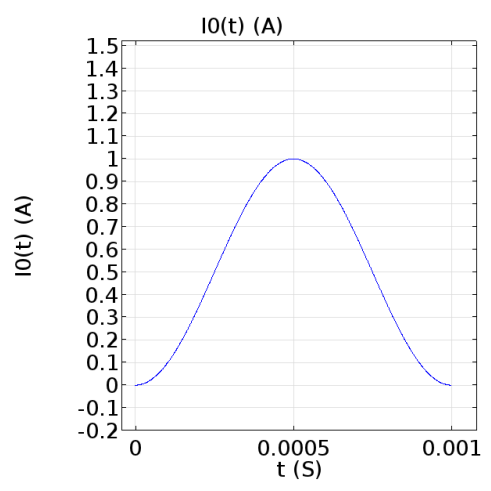

Figure 1. The transmission coil excitation function.

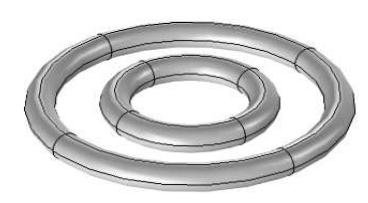

$$
y \stackrel{z}{2}-x
$$

Figure 2. Geometry configuration of probe coils; the inductive in the external part and the receiver is the internal part.

Solution of equations (3) can be achieved by means of the FEM. This was performed in the present work by using Comsol Multiphysics. Here, use was made of the 
AC/DC Module which has the capacity of simulating electromagnetic problems.

Two electrical quantities used to characterise the state of the testing coil are expressed as ohmic resistance $R$ and inductance $L$. Under harmonic excitation, the reactance is related to the inductance by the following relation

$$
Z_{L}=2 \pi f L
$$

with

$$
L=L_{s}+L_{m}
$$

where $f$ is the frequency of exciting current, while $L$ is the combination of self inductance of the coil $L_{s}$ and mutual inductance $L_{m}$.

Neglecting the capacitance, the coil impedance is given by

$$
Z=\sqrt{R^{2}+4 \pi^{2} f^{2} L^{2}}
$$

By fixing the testing frequency spectrum and lift-off of the probe, only the conductivity of the sample influences the receiver coil impedance. If an object with certain electrical conductivity is put close to this coil, the inductive magnetic field is modified which is indicated by the change of the impedance. The coil will undergo change of impedance when a defect is present in the sample. By tracking variations of the impedance, detection of defects can then be readily monitored.

\section{Results and discussion}

Let's consider a rectangular plate made from CFPR composite, Figure 3. The plate has the length $L=800 \mathrm{~mm}$, the width $\ell=400 \mathrm{~mm}$ and depth $h=10 \mathrm{~mm}$. The plate material characteristics are $\sigma=95 \mathrm{~S} / \mathrm{m}$ and $\mu_{r}=1 H / m$. A defect having the form of an open parallelepiped cavity that has sides parallel to those of the plate is assumed to be present at the centre of the plate. Its dimensions are taken to be given by $L_{d}=20 \mathrm{~mm}, \ell_{d}=20 \mathrm{~mm}$ and $h_{d}=5 \mathrm{~mm}$
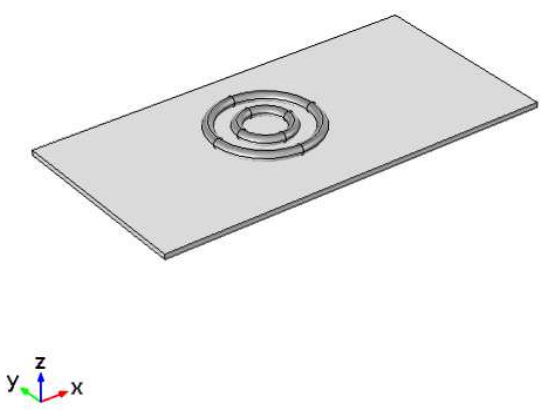

Figure 3. The test plate sample model geometry.
The inductive coil is assumed to be made from copper for which $\sigma^{\prime}=6 \times 10^{7} \mathrm{~S} / \mathrm{m}$ and $\mu_{r}^{\prime}=1 \mathrm{H} / \mathrm{m}$. The coil base is circular with radius $5 \mathrm{~mm}$. This coil is assumed to be formed by 1000 whorls having the section area $a_{c}=5.7810^{-5} \mathrm{~m}^{2}$. It is assumed to be excited by a pulsed current of the form

$$
I(t)=\frac{1}{2}[1+\cos (2 \pi(t-T / 2) / T)] \text { for } t \in[0, T]
$$

The sensor coil is assumed also to be made from copper. The base of receiver coil is circular with radius $2.5 \mathrm{~mm}$.

A FEM model was developed for the system composed from the test plate, the probe and the ambient air. The mesh was generated automatically by using free tetrahedral elements under Comsol. The maximum size of mesh was fixed at $20 \mathrm{~mm}$ for the plate and $10 \mathrm{~mm}$ for the coils.

As the excitation and receiver coil are coupled to the test specimen through air according to equations (2), the environmental air which a purely propagative medium should also be meshed. Because meshing an infinite volume is not possible, it is necessary to specify a finite volume to mesh and solve for it. In the present study, the whole probe-coil setup was placed at the centre of a cube having $2000 \mathrm{~mm}$ edge, see Figure 4.

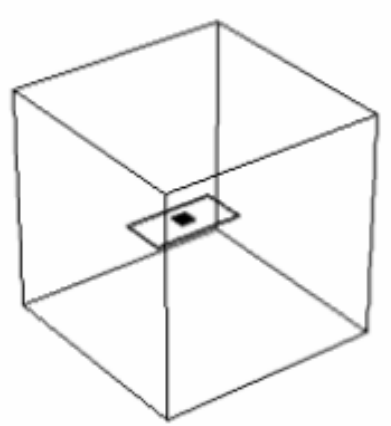

Figure 4. The complete model of the EC probe setup and test specimen.

To avoid reflection that is susceptible to take place in a finite volume domain, the default boundary condition corresponding to magnetic insulation is used at the cube boundaries. This condition forces the field to be tangential to the exterior boundaries. The air electric conductivity is fixed at $\sigma_{\text {air }}=1 S / \mathrm{m}$. The pulse frequency is fixed at $f=1000 \mathrm{~Hz}$ which correspond to $T=1 m s$ in Eq. 7 .

Both the inductive and the sensor coil are placed at the same distance from the top surface of the plate assumed to be planar and perfectly smooth. The air gap separating the coils from the inspected plate was fixed at $d=15 \mathrm{~mm}$. The EC intensity in the receiver coil was obtained by FEM post-processing.

Figures 5 and 6 give the obtained density of eddycurrents at time $t=2 T$ respectively in the defect free plate and the plate with the presence of defect, while the probe 
occupies the same location (over the defect) during the B-scan procedure.

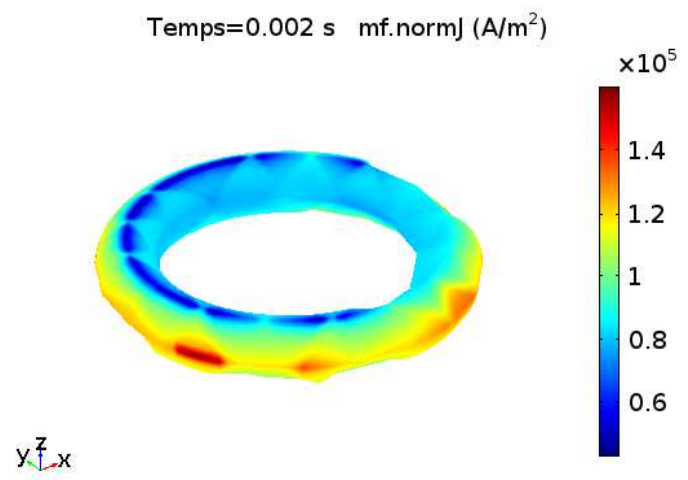

Figure 5. Current density in the receiver coil (plate without defect) at instant $t=2 T$.

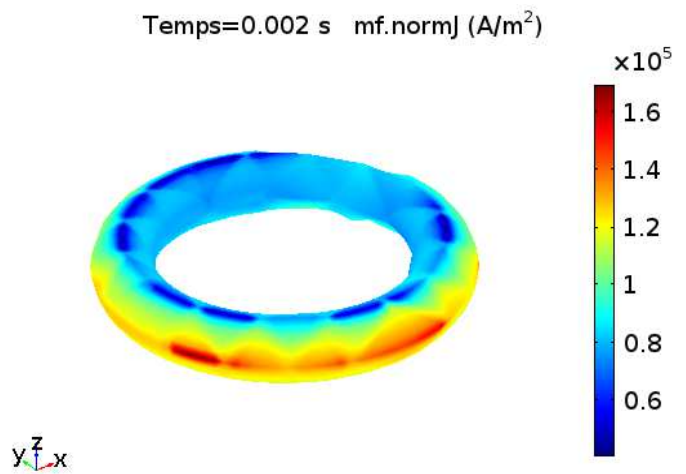

Figure 6. Current density in the receiver coil (plate with defect) at instant $t=2 T$.

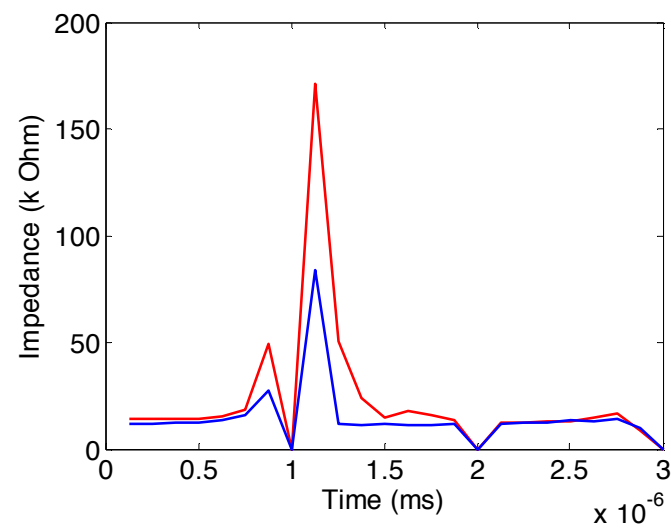

Figure 7. Comparison of impedance of receiver coil between the free defect case and the defected plate.

Figure 7 gives a comparison, in terms of impedances time evolution for the receiver coil between the case of defect free-plate and flawed plate.

Figure 7 shows that the defect induces a signature on the time variation of coil impedance that can be monitored in order to sense the defect. A defect indicator can like this be defined in order to proceed to defect detection in metallic parts. It should be noticed that while working with harmonic like excitation, this signature was very poor and cannot be readily used to detect the defect.

\section{Conclusions}

In this paper, a transmitter/receiver probe has been proposed to perform detection of defects appearing in composites parts made from Carbon Fiber Reinforced Plastics. Transient pulsed eddy-current based technique was implemented by using COMSOL Multiphysics software packages. Considering a B-scan inspection procedure, detection of small flaws was demonstrated in situations where conventional stationary harmonic eddycurrent based technique fails to manage any detection. This was achieved through monitoring impedance variations of the receiver coil.

Further studies are needed in order to optimise detection as function of the central work frequency and probe geometric characteristics.

\section{References}

1. L. Rosado, T.G. Santos, P.M. Ramos, P. Vilac, M. Piedade, NDT\&E International 51 (2013)

2. M. Gerhard, L. Rolf, K. Ole, Compos Sci Technol 61 (2001)

3. T.W. Krause, V.K. Babbar, P.R. Underhill, AIP Conference Proceedings 1581 (2014)

4. D.P.R. Desjardins, T.W. Krause, N. Gauthier, Nondestructive Testing and Evaluation International 60 (2013)

5. M. Ur Rahman, Optimization of in-line defect detection by eddy current techniques (PhD Thesis, Kassel University, Germany, 2010)

6. A. Rosell, G. Persson, International Journal of Fatigue 41 (2012) 